\title{
On the Finite Dimensional Laws of Threshold GARCH Processes
}

\author{
Esmeralda Gonçalves, Joana Leite, Nazaré Mendes-Lopes
}

\begin{abstract}
In this paper we establish bounds for the finite dimensional laws of a threshold GARCH process, $X$, with generating process $Z$. In this class of models the conditional standard deviation has different reactions according to the sign of past values of the process. So, we firstly find lower and upper bounds for the law of $\left(X_{1}^{+},-X_{1}^{+}, \ldots, X_{n}^{+},-X_{n}^{+}\right)$, in certain regions of $\mathbb{R}^{2 n}$, and use them to find bounds of the law of $\left(X_{1}, \ldots, X_{n}\right)$. Some of these bounds only depend on the parameters of the model and on the distribution function of the independent generating process, $Z$. An application of these bounds to control charts for time series is presented.
\end{abstract}

\section{Introduction}

As the true theoretical law of conditional heteroskedastic models is difficult to find, the most part of the analysis undertaken for these models is dedicated to the study of properties or probabilistic summaries of those laws. The use of these models, for instance within the quality control theory, needs the assessment of the probability of certain regions depending on the process. So, contrary to that trend, Pawlak and Schmid [5], Gonçalves, Leite and Mendes-Lopes [1] and Gonçalves and MendesLopes [3] develop studies to find bounds for the finite dimensional laws of certain transformations of ARCH and TGARCH processes, respectively, here denoted $X$.

E. Gonçalves

CMUC, Department of Mathematics, University of Coimbra, 3001-454 Coimbra, Portugal

e-mail: esmerald@mat.uc.pt

J. Leite

Institute of Accounting and Administration of Coimbra, Quinta Agrícola, Bencanta, 3040-316,

Coimbra, Portugal, e-mail: jleite@iscac.pt

N. Mendes-Lopes

CMUC, Department of Mathematics, University of Coimbra, 3001-454 Coimbra, Portugal

e-mail: nazare@mat.uc.pt 
These bounds are expressed in terms of the distribution function of the independent generating process, $Z$, and it becomes clear that the marginal law of $X$ is, in certain regions, strongly controlled by that of the process $Z$. This fact is rather relevant as we know that these laws have in general quite different characteristics; for example, the marginal law of $X$ is leptocurtic even if it does not happen with that of the independent generating process. The application in time series of this kind of bounds on control charts with symmetric control limits has been explored in the literature (Severin and Schmid [6], Gonçalves, Leite and Mendes-Lopes [2]), due to the relevance of the target process distribution in the alarm signal definition as it makes possible to analyze the probability of having the process out of control in a given moment.

For a real stochastic process $X=\left(X_{t}, t \in \mathbb{Z}\right)$ let us define $X_{t}^{+}=X_{t} \mathbb{I}_{\left\{X_{t} \geq 0\right\}}, X_{t}^{-}=$ $X_{t} \mathbb{I}_{\left\{X_{t}<0\right\}}$ and $\underline{X}_{t}$ the sigma field generated by $X_{t}, X_{t-1}, \ldots$

The process $X$ follows a generalized threshold auto-regressive conditionally heteroskedastic model with orders $p$ and $q, \operatorname{TGARCH}(p, q)$, if for real constants $\alpha_{0}>0, \alpha_{i} \geq 0, \beta_{i} \geq 0, \gamma_{j} \geq 0,(i=1, \ldots, q, j=1, \ldots, p)$ and a sequence of independent and identically distributed real random variables, $\left(Z_{t}, t \in \mathbb{Z}\right)$, with zero mean, unit variance and $Z_{t}$ independent of $\underline{X}_{t-1}$ we have, for every $t \in \mathbb{Z}$,

$$
\left\{\begin{array}{l}
X_{t}=\sigma_{t} Z_{t} \\
\sigma_{t}=\alpha_{0}+\sum_{i=1}^{q} \alpha_{i} X_{t-i}^{+}-\sum_{i=1}^{q} \beta_{i} X_{t-i}^{-}+\sum_{j=1}^{p} \gamma_{j} \sigma_{t-j} .
\end{array}\right.
$$

The process $Z=\left(Z_{t}, t \in \mathbb{Z}\right)$ is called the generating process of $X$. If $\gamma_{j}=0, j=$ $1, \ldots, p$, we say that $X$ follows a $\operatorname{TARCH}(q)$ model.

The main characteristic of threshold conditionally heteroskedastic models is the fact that they allow to take into account different reactions in the volatility according to the sign of the process values even for values with the same absolute size. So, these models capture the so-called leverage effect very common in financial time series of daily returns (Malmsten and Terasvirta [4]). A not so very common, but yet still present characteristic in some daily returns series is skewness, positive in some cases and negative in others (Taylor [7]). For these financial series, only control charts designed with asymmetric control limits are appropriate. So, following the ideas of Gonçalves and Mendes-Lopes [3] for the finite dimensional laws of the absolute value process of a TGARCH model, we firstly establish in Section 2 lower and upper bounds for the law of $\left(X_{1}^{+},-X_{1}^{-}, \ldots, X_{n}^{+},-X_{n}^{-}\right)$, in certain regions of $\mathbb{R}^{2 n}$. A relationship between the finite dimensional laws of the process $X$ and the corresponding laws of $|X|$ and $\left(X^{+}, X^{-}\right)$processes is obtained in Section 3 from which we bound the distribution function of $\left(X_{1}, \ldots, X_{n}\right)$. Some of these bounds only depend on the parameters of the model and on the distribution function of the error process $Z$. These new results on the distribution function of $\left(X_{1}^{+},-X_{1}^{-}, \ldots, X_{n}^{+},-X_{n}^{-}\right)$, are essential to generalize the study developed in Gonçalves, Leite and MendesLopes [2] to control charts with asymmetric limits. A preliminary contribution for the evaluation of the run length of this kind of control charts is presented in Section 4. In the last Section some concluding remarks are included. 
2 Bounds for the Distribution Function of $\left(X_{1}^{+},-X_{1}^{-}, \ldots, X_{n}^{+},-X_{n}^{-}\right)$

Let $X=\left(X_{t}, t \in \mathbb{Z}\right)$ be a TGARCH$(p, q)$ process. In the following, the distribution function of $Z_{t}$ is denoted by $F_{Z}$ and, if the law of $Z_{t}$ is absolutely continuous, $f_{Z}$ denotes its density of probability function. We also consider the conventions $\sum_{i=1}^{0}()=$.0 and, for $n<q+1, \prod_{t=q+1}^{n}()=$.1 .

If the law of $Z_{t}$ is diffuse, we have the following upper bound for the distribution function of $\left(X_{1}^{+},-X_{1}^{-}, \ldots, X_{n}^{+},-X_{n}^{-}\right)$:

$$
F_{\left(X_{1}^{+},-X_{1}^{-}, \ldots, X_{n}^{+},-X_{n}^{-}\right)}\left(x_{1}, x_{1}^{*}, \ldots, x_{n}, x_{n}^{*}\right) \leq \prod_{t=1}^{n}\left[F_{Z}\left(\frac{x_{t}}{\theta}\right)-F_{Z}\left(-\frac{x_{t}^{*}}{\theta}\right)\right]
$$

for every $\left(x_{1}, x_{1}^{*}, \ldots, x_{n}, x_{n}^{*}\right) \in\left[0,+\infty\left[^{2 n}\right.\right.$ and where $\theta=\alpha_{0}\left(1+\sum_{j=1}^{p} \gamma_{j}\right)$.

This result follows easily taking into account the equality

$$
\begin{aligned}
& F_{\left(X_{1}^{+},-X_{1}^{-}, \ldots, X_{n}^{+},-X_{n}^{-}\right)}\left(x_{1}, x_{1}^{*}, \ldots, x_{n}, x_{n}^{*}\right) \\
& =P\left(-\frac{x_{1}^{*}}{\sigma_{1}} \leq Z_{1} \leq \frac{x_{1}}{\sigma_{1}}, \ldots,-\frac{x_{n}^{*}}{\sigma_{n}} \leq Z_{n} \leq \frac{x_{n}}{\sigma_{n}}\right),
\end{aligned}
$$

the inequality $\sigma_{t} \geq \alpha_{0}+\sum_{j=1}^{p} \gamma_{j} \sigma_{t-j}$ as well as the independence and identical law of $Z_{1}, \ldots, Z_{n}$.

The determination of a lower bound of $F_{\left(X_{1}^{+},-X_{1}^{-}, \ldots, X_{n}^{+},-X_{n}^{-}\right.}$is more complex and it is subject to more restrictive hypotheses. The following theorem states a result for TARCH(q) and TGARCH $(1,1)$ models. The general case of TGARCH (p,q) models is studied in an analogous way, with increased calculations complexity. Let us define the real function $h(x)=x f_{Z}^{\prime}(x)+2 f_{Z}(x), x \in \mathbb{R}$.

Theorem 2.1. Let $X=\left(X_{t}, t \in \mathbb{Z}\right)$ be a stationary $\operatorname{TGARCH}(p, q)$ process, with variance $\sigma_{X}^{2}$, such that the law of $Z_{t}$ is absolutely continuous with a differentiable density of probability. For $\left(x_{1}, x_{1}^{*}, \ldots, x_{n}, x_{n}^{*}\right) \in\left[0,+\infty{ }^{2 n}\right.$ such that

$$
\forall y \geq 0, \quad g(y)=x_{t} h\left(\frac{x_{t}}{\alpha_{0}+y}\right)+x_{t}^{*} h\left(-\frac{x_{t}^{*}}{\alpha_{0}+y}\right) \geq 0,
$$

where

$$
t= \begin{cases}1, \ldots, \min \{q, n\}, & \text { if } p=0 \\ 1, \ldots, n, & \text { if } p=1\end{cases}
$$

we have:

a) if $p=0$ and $1 \leq q<n$,

$$
\begin{aligned}
& F_{\left(X_{1}^{+},-X_{1}^{-}, \ldots, X_{n}^{+},-X_{n}^{-}\right)}\left(x_{1}, x_{1}^{*}, \ldots, x_{n}, x_{n}^{*}\right) \\
& \geq \prod_{t=1}^{q}\left[F_{Z}\left(\frac{x_{t}}{u_{t}}\right)-F_{Z}\left(-\frac{x_{t}^{*}}{u_{t}}\right)\right] \prod_{t=q+1}^{n}\left[F_{Z}\left(\frac{x_{t}}{v_{t}}\right)-F_{Z}\left(-\frac{x_{t}^{*}}{v_{t}}\right)\right]
\end{aligned}
$$


where $u_{t}=\alpha_{0}+\sum_{i=1}^{t-1}\left(\alpha_{i} x_{t-i}+\beta_{i} x_{t-i}^{*}\right)+E\left(X_{t}^{+}\right) \sum_{i=t}^{q}\left(\alpha_{i}+\beta_{i}\right), t=1, \ldots, q$, and $v_{t}=\alpha_{0}+\sum_{i=1}^{q}\left(\alpha_{i} x_{t-i}+\beta_{i} x_{t-i}^{*}\right), t=q+1, \ldots, n$;

b) if $p=0$ and $q \geq n \geq 1$,

$$
F_{\left(X_{1}^{+},-X_{1}^{-}, \ldots, X_{n}^{+},-X_{n}^{-}\right)}\left(x_{1}, x_{1}^{*}, \ldots, x_{n}, x_{n}^{*}\right) \geq \prod_{t=1}^{n}\left[F_{Z}\left(\frac{x_{t}}{u_{t}}\right)-F_{Z}\left(-\frac{x_{t}^{*}}{u_{t}}\right)\right]
$$

where $u_{t}=\alpha_{0}+\sum_{i=1}^{t-1}\left(\alpha_{i} x_{t-i}+\beta_{i} x_{t-i}^{*}\right)+E\left(X_{t}^{+}\right) \sum_{i=t}^{q}\left(\alpha_{i}+\beta_{i}\right), t=1, \ldots, n$;

c) if $p=1$ and $q=1$,

$$
F_{\left(X_{1}^{+},-X_{1}^{-}, \ldots, X_{n}^{+},-X_{n}^{-}\right)}\left(x_{1}, x_{1}^{*}, \ldots, x_{n}, x_{n}^{*}\right) \geq \prod_{t=1}^{n}\left[F_{Z}\left(\frac{x_{t}}{w_{t}}\right)-F_{Z}\left(-\frac{x_{t}^{*}}{w_{t}}\right)\right]
$$

where $w_{t}=\alpha_{0} \sum_{j=1}^{t-1} \gamma_{1}^{j-1}+\sum_{i=1}^{t-1} \gamma_{1}^{j-1}\left(\alpha_{1} x_{t-j}+\beta_{1} x_{t-j}^{*}\right)+\gamma_{1}^{t-1} E\left(\sigma_{1}\right), t=1, \ldots, n$.

Proof. We present the proof for the situation considered in a), $p=0$ and $1 \leq q \leq n$. Let $\left(x_{1}, x_{1}^{*}, \ldots, x_{n}, x_{n}^{*}\right) \in\left[0,+\infty\left[2 n\right.\right.$. We note that if $X_{1}^{+} \leq x_{1},-X_{1}^{-} \leq x_{1}^{*}, \ldots, X_{n}^{+} \leq$ $x_{n},-X_{n}^{-} \leq x_{n}^{*}$ then

(i) for $t \in\{2, \ldots, q\}, \sigma_{t} \leq \alpha_{0}+\sum_{i=1}^{t-1}\left(\alpha_{i} x_{t-i}+\beta_{i} x_{t-i}^{*}\right)+\sum_{i=t}^{q}\left(\alpha_{i} X_{t-i}^{+}-\beta_{i} X_{t-i}^{-}\right)=S_{t}$;

(ii) for $t=1, \sigma_{1}=\alpha_{0}+\sum_{i=1}^{q}\left(\alpha_{i} X_{1-i}^{+}-\beta_{i} X_{1-i}^{-}\right)=S_{1}$;

(iii) and for $t \in\{q+1, \ldots, n\}, \sigma_{t}=\alpha_{0}+\sum_{i=1}^{q}\left[\alpha_{i} X_{t-i}^{+}+\beta_{i}\left(-X_{t-i}^{-}\right)\right]$

$$
\leq \alpha_{0}+\sum_{i=1}^{q}\left(\alpha_{i} x_{t-i}+\beta_{i} x_{t-i}^{*}\right)=v_{t} .
$$

So,

$$
\begin{aligned}
& F_{\left(X_{1}^{+},-X_{1}^{-}, \ldots, X_{n}^{+},-X_{n}^{-}\right)}\left(x_{1}, x_{1}^{*}, \ldots, x_{n}, x_{n}^{*}\right) \\
& \quad=P\left(-\frac{x_{t}^{*}}{\sigma_{t}} \leq Z_{t} \leq \frac{x_{t}}{\sigma_{t}}, t=1, \ldots, q,-\frac{x_{t}^{*}}{\sigma_{t}} \leq Z_{t} \leq \frac{x_{t}}{\sigma_{t}}, t=q+1, \ldots, n\right) \\
& \geq P\left(-\frac{x_{t}^{*}}{S_{t}} \leq Z_{t} \leq \frac{x_{t}}{S_{t}}, t=1, \ldots, q,-\frac{x_{t}^{*}}{v_{t}} \leq Z_{t} \leq \frac{x_{t}}{v_{t}}, t=q+1, \ldots, n\right) .
\end{aligned}
$$

As $S_{t}$ is $\underline{X}_{0}$-measurable $(t=1, \ldots, q), v_{t}$ is non random $(t=q+1, \ldots, n), Z_{t}$ is independent of $\underline{X}_{t-1}$ and $\underline{X}_{0} \subset \underline{X}_{t-1}, Z_{1}, \ldots, Z_{n}$ are independent and identically distributed and absolutely continuous, we get, using the properties of the mean and conditional mean,

$$
\begin{aligned}
& P\left(-\frac{x_{t}^{*}}{S_{t}} \leq Z_{t} \leq \frac{x_{t}}{S_{t}}, t=1, \ldots, q,-\frac{x_{t}^{*}}{v_{t}} \leq Z_{t} \leq \frac{x_{t}}{v_{t}}, t=q+1, \ldots, n\right) \\
& \quad=E\left[P\left(-\frac{x_{t}^{*}}{S_{t}} \leq Z_{t} \leq \frac{x_{t}}{S_{t}}, t=1, \ldots, q,-\frac{x_{t}^{*}}{v_{t}} \leq Z_{t} \leq \frac{x_{t}}{v_{t}}, t=q+1, \ldots, n \mid \underline{X}_{0}\right)\right] \\
& =\prod_{t=1}^{q} E\left[F_{Z}\left(\frac{x_{t}}{S_{t}}\right)-F_{Z}\left(-\frac{x_{t}^{*}}{S_{t}}\right)\right] \prod_{t=q+1}^{n} E\left[F_{Z}\left(\frac{x_{t}}{v_{t}}\right)-F_{Z}\left(-\frac{x_{t}^{*}}{v_{t}}\right)\right] .
\end{aligned}
$$


For $t$ arbitrarily fixed in $\{1, \ldots, q\}$, let us consider the function $R_{t}:[0,+\infty[\longrightarrow$ $[-1,1]$ defined by $R_{t}(y)=F_{Z}\left(\frac{x_{t}}{\alpha_{0}+y}\right)-F_{Z}\left(-\frac{x_{t}^{*}}{\alpha_{0}+y}\right)$. We have

$$
\frac{d^{2} R_{t}}{d y^{2}}(y)=\frac{1}{\left(\alpha_{0}+y\right)^{3}} g(y) \text {. }
$$

So, for every $t \in\{1, \ldots, q\}$, if $g(y) \geq 0$, for every $y \geq 0$, then $R_{t}$ is a convex function. In these conditions we may apply Jensen inequality and we obtain

$$
\begin{aligned}
\prod_{t=1}^{q} E & {\left[F_{Z}\left(\frac{x_{t}}{S_{t}}\right)-F_{Z}\left(-\frac{x_{t}^{*}}{S_{t}}\right)\right] \prod_{t=q+1}^{n} E\left[F_{Z}\left(\frac{x_{t}}{v_{t}}\right)-F_{Z}\left(-\frac{x_{t}^{*}}{v_{t}}\right)\right] } \\
& \geq \prod_{t=1}^{q}\left[F_{Z}\left(\frac{x_{t}}{E\left(S_{t}\right)}\right)-F_{Z}\left(-\frac{x_{t}^{*}}{E\left(S_{t}\right)}\right)\right] \prod_{t=q+1}^{n}\left[F_{Z}\left(\frac{x_{t}}{v_{t}}\right)-F_{Z}\left(-\frac{x_{t}^{*}}{v_{t}}\right)\right]
\end{aligned}
$$

and so

$$
\begin{aligned}
& F_{\left(X_{1}^{+},-X_{1}^{-}, \ldots, X_{n}^{+},-X_{n}^{-}\right)}\left(x_{1}, x_{1}^{*}, \ldots, x_{n}, x_{n}^{*}\right) \\
& \geq \prod_{t=1}^{q}\left[F_{Z}\left(\frac{x_{t}}{u_{t}}\right)-F_{Z}\left(-\frac{x_{t}^{*}}{u_{t}}\right)\right] \prod_{t=q+1}^{n}\left[F_{Z}\left(\frac{x_{t}}{v_{t}}\right)-F_{Z}\left(-\frac{x_{t}^{*}}{v_{t}}\right)\right],
\end{aligned}
$$

taking into account that $E\left(S_{t}\right)=u_{t}$.

Remark. We point out that $E\left(X_{t}^{+}\right)$and $E\left(\sigma_{1}\right)$ only depend on the coefficients of the model and on $Z$ law. In fact, as $X$ is second order stationary, it is also strictly stationary; so, $E\left(\sigma_{t}\right)$ is independent of $t$ and we obtain

$$
E\left(X_{t}^{+}\right)=E\left(\sigma_{t}\right) E\left(Z_{t}^{+}\right)=\frac{\alpha_{0}}{1-E\left(Z_{t}^{+}\right) \sum_{i=t}^{q}\left(\alpha_{i}+\beta_{i}\right)-\sum_{i=t}^{p} \gamma_{i}} E\left(Z_{t}^{+}\right) .
$$

The result presented is valid for a large class of probability laws of the process $Z$. In order to get some insight on the sets where the lower bounds obtained for the distribution function of $\left(X_{1}^{+},-X_{1}^{-}, \ldots, X_{n}^{+},-X_{n}^{-}\right)$are valid, related to the positivity of the $g$ function, we analyze the positivity of the $h$ function considering two distributions particularly useful in the applications.

Example 2.1. For $Z_{t}$ distributed according to the standard Gaussian law, we have $h(x)=\frac{1}{\sqrt{2 \pi}} f_{Z}(x)\left[-x^{2}+2\right], x \in \mathbb{R}$, and so $h(x) \geq 0$ if $x \in[-\sqrt{2}, \sqrt{2}]$.

Example 2.2. We consider now that $Z_{t}$ follows a centered and reduced Laplace law, that is, $Z_{t}$ is absolutely continuous with density $f_{Z}(y)=\frac{\sqrt{2}}{2} \exp \left(-\frac{\sqrt{2}}{2}|y|\right), y \in \mathbb{R}$. We obtain, for $y>0, h(y)=f_{Z}(y)\left(-\frac{\sqrt{2}}{2} y+2\right)$ and $h(y) \geq 0$ if $y \leq 2 \sqrt{2}$. 
3 Bounds for the Distribution Function of $\left(X_{1}, \ldots, X_{n}\right)$

As a probabilistic application of the results developed in Section 2 we analyze now some bounds of the distribution function of $\left(X_{1}, \ldots, X_{n}\right)$ in the regions $] 0,+\infty\left[{ }^{n}\right.$ and ]$-\infty, 0\left[{ }^{n}\right.$. Let us consider $\theta=\alpha_{0}\left(1+\sum_{j=1}^{p} \gamma_{j}\right)$.

If $\left.\left(x_{1}, \ldots, x_{n}\right) \in\right] 0,+\infty\left[^{n}\right.$ we easily conclude that

$$
F_{\left(X_{1}, \ldots, X_{n}\right)}\left(x_{1}, \ldots, x_{n}\right) \leq \prod_{t=1}^{n} F_{Z}\left(\frac{x_{t}}{\theta}\right) .
$$

A lower bound for $F_{\left(X_{1}, \ldots, X_{n}\right)}\left(x_{1}, \ldots, x_{n}\right)$ is found as a consequence of the following theorem.

Theorem 3.1. Let $X=\left(X_{t}, t \in \mathbb{Z}\right)$ be a $\operatorname{TGARCH}(p, q)$ process such that the law of $Z_{t}$ is absolutely continuous. Then, for every $\left.\left(x_{1}, \ldots, x_{n}\right) \in\right] 0,+\infty\left[{ }^{n}\right.$,

$$
\begin{aligned}
& F_{\left(X_{1}, \ldots, X_{n}\right)}\left(x_{1}, \ldots, x_{n}\right) \geq \\
& \quad \geq\left[F_{Z}(0)\right]^{n}+\sum_{t=1}^{n} F_{\left(X_{1}^{+},-X_{1}^{-}, \ldots, X_{t}^{+},-X_{t}^{-}\right)}\left(x_{1}, 0, x_{2}, 0, \ldots, x_{t}, 0\right)\left[F_{Z}(0)\right]^{n-t} .
\end{aligned}
$$

Proof. We have

$$
\begin{aligned}
& F_{\left(X_{1}, \ldots, X_{n}\right)}\left(x_{1}, \ldots, x_{n}\right)=P\left(X_{1} \leq x_{1}, X_{t} \leq x_{t}, t=2, \ldots, n\right) \\
& =P\left(0 \leq X_{1} \leq x_{1}, X_{t} \leq x_{t}, t=2, \ldots, n\right)+P\left(X_{1}<0, X_{t} \leq x_{t}, t=2, \ldots, n\right) \\
& =P\left(0 \leq X_{1} \leq x_{1}, 0 \leq X_{2} \leq x_{2}, X_{t} \leq x_{t}, t=3, \ldots, n\right)+ \\
& \quad+P\left(0 \leq X_{1} \leq x_{1}, X_{2}<0, X_{t} \leq x_{t}, t=3, \ldots, n\right)+ \\
& \quad+P\left(X_{1}<0,0 \leq X_{2} \leq x_{2}, X_{t} \leq x_{t}, t=3, \ldots, n\right)+ \\
& \quad+P\left(X_{1}<0, X_{2}<0, X_{t} \leq x_{t}, t=3, \ldots, n\right) .
\end{aligned}
$$

Repeating this reasoning we obtain

$$
\begin{aligned}
F_{\left(X_{1}, \ldots, X_{n}\right)}\left(x_{1}, \ldots, x_{n}\right) \\
\geq P\left(0 \leq X_{t} \leq x_{t}, t=1, \ldots, n\right)+P\left(X_{t}<0, t=1, \ldots, n\right) \\
\quad+\sum_{t=1}^{n-1} P\left(0 \leq X_{i} \leq x_{i}, i=1, \ldots, t, X_{i}<0, i=t+1, \ldots, n\right) .
\end{aligned}
$$

But

$$
\begin{aligned}
P\left(0 \leq X_{t} \leq x_{t}, t=1, \ldots, n\right) & =P\left(X_{t}^{+} \leq x_{t},-X_{t}^{-} \leq 0, t=1, \ldots, n\right) \\
& =F_{\left(X_{1}^{+},-X_{1}^{-}, \ldots, X_{n}^{+},-X_{n}^{-}\right)}\left(x_{1}, 0, \ldots, x_{n}, 0\right) .
\end{aligned}
$$

Further, as $P\left(X_{t}<0, t=1, \ldots, n\right)=\left[F_{Z}(0)\right]^{n}$ and $Z_{i}$ is independent of $\underline{X}_{i-1}, i \in$ $\{t+1, \ldots, n\}$, then 


$$
\begin{aligned}
P(0 & \left.\leq X_{i} \leq x_{i}, i=1, \ldots, t, X_{i}<0, i=t+1, \ldots, n\right) \\
& =F_{\left(X_{1}^{+},-X_{1}^{-}, \ldots, X_{t}^{+},-X_{t}^{-}\right)}\left(x_{1}, 0, \ldots, x_{t}, 0\right)\left[F_{Z}(0)\right]^{n-t} .
\end{aligned}
$$

In this way we obtain

$$
\begin{aligned}
& F_{\left(X_{1}, \ldots, X_{n}\right)}\left(x_{1}, \ldots, x_{n}\right) \geq F_{\left(X_{1}^{+},-X_{1}^{+}, \ldots, X_{n}^{+},-X_{n}^{+}\right)}\left(x_{1}, 0, \ldots, x_{n}, 0\right) \\
& \quad+\left[F_{Z}(0)\right]^{n}+\sum_{t=1}^{n-1} F_{\left(X_{1}^{+},-X_{1}^{-}, \ldots, X_{t}^{+},-X_{t}^{-}\right)}\left(x_{1}, 0, \ldots, x_{t}, 0\right)\left[F_{Z}(0)\right]^{n-t} .
\end{aligned}
$$

Remark. A lower bound of $F_{\left(X_{1}, \ldots, X_{n}\right)}\left(x_{1}, \ldots, x_{n}\right)$ depending only on the coefficients of the model and on the distribution function of $Z$ may be obtained taking into account the results of the previous Section. In fact, if we consider, for example, that $X$ follows a TARCH $(q)$ model (with $q \leq n$ ) we get for $1 \leq t \leq n$ and under the conditions of Theorem 1 ,

$$
\begin{aligned}
\left.F_{\left(X_{1}^{+},-X_{1}^{-}, \ldots, X_{t}^{+},-\right.} X_{t}^{-}\right)\left(x_{1}, 0, \ldots, x_{t}, 0\right) \geq \\
\quad \geq \begin{cases}\prod_{j=1}^{q}\left[F_{Z}\left(\frac{x_{j}}{u_{j}}\right)-F_{Z}(0)\right] \prod_{j=q+1}^{t}\left[F_{Z}\left(\frac{x_{j}}{v_{j}}\right)-F_{Z}(0)\right], & 1 \leq q<t, \\
\prod_{j=1}^{t}\left[F_{Z}\left(\frac{x_{j}}{u_{j}}\right)-F_{Z}(0)\right], & t \leq q,\end{cases}
\end{aligned}
$$

where $u_{j}=\alpha_{0}+\sum_{i=1}^{j-1} \alpha_{i} x_{j-i}+E\left(X_{t}^{+}\right) \sum_{i=j}^{q}\left(\alpha_{i}+\beta_{i}\right)$ and $v_{j}=\alpha_{0}+\sum_{i=1}^{q} \alpha_{i} x_{j-i}$. So, for a process $X$ following a TARCH$(q)$ model we have, if $\left.\left(x_{1}, \ldots, x_{n}\right) \in\right] 0,+\infty{ }^{n}$,

$$
\begin{aligned}
& F_{\left(X_{1}, \ldots, X_{n}\right)}\left(x_{1}, \ldots, x_{n}\right) \\
& \geq\left[F_{Z}(0)\right]^{n}+\sum_{t=1}^{q}\left\{\left[F_{Z}(0)\right]^{n-t} \prod_{j=1}^{t}\left[F_{Z}\left(\frac{x_{j}}{u_{j}}\right)-F_{Z}(0)\right]\right\} \\
& \quad+\sum_{t=q+1}^{n}\left\{\left[F_{Z}(0)\right]^{n-t} \prod_{j=1}^{q}\left[F_{Z}\left(\frac{x_{j}}{u_{j}}\right)-F_{Z}(0)\right] \prod_{j=q+1}^{t}\left[F_{Z}\left(\frac{x_{j}}{v_{j}}\right)-F_{Z}(0)\right]\right\} .
\end{aligned}
$$

Let us now consider $\left.\left(x_{1}, \ldots, x_{n}\right) \in\right]-\infty, 0\left[{ }^{n}\right.$. We get

$$
F_{\left(X_{1}, \ldots, X_{n}\right)}\left(x_{1}, \ldots, x_{n}\right) \geq P\left(Z_{1} \leq \frac{x_{1}}{\theta}, \ldots, Z_{n} \leq \frac{x_{n}}{\theta}\right)=\prod_{t=1}^{n} P\left(Z_{t} \leq \frac{x_{t}}{\theta}\right)=\prod_{t=1}^{n} F_{Z}\left(\frac{x_{t}}{\theta}\right) .
$$

Otherwise, for $\left(x_{1}, \ldots, x_{n}\right) \in\left[0,+\infty\left[^{n}\right.\right.$, an upper bound of $F_{\left(X_{1}, \ldots, X_{n}\right)}\left(-x_{1}, \ldots,-x_{n}\right)$ can be obtained in terms of $F_{\left(X_{1}, \ldots, X_{n}\right)}\left(x_{1}, \ldots, x_{n}\right)$ and of $F_{\left(\left|X_{1}\right|, \ldots,\left|X_{n}\right|\right)}\left(x_{1}, \ldots, x_{n}\right)$, as stated in the next theorem.

Theorem 3.2. Let $X=\left(X_{t}, t \in \mathbb{Z}\right)$ be a $\operatorname{TGARCH}(p, q)$ process such that the law of $Z_{t}$ is diffuse. Then, for every $\left(x_{1}, \ldots, x_{n}\right) \in\left[0,+\infty\left[^{n}\right.\right.$, 


$$
F_{\left(X_{1}, \ldots, X_{n}\right)}\left(-x_{1}, \ldots,-x_{n}\right) \leq F_{\left(X_{1}, \ldots, X_{n}\right)}\left(x_{1}, \ldots, x_{n}\right)-F_{\left(\left|X_{1}\right|, \ldots,\left|X_{n}\right|\right)}\left(x_{1}, \ldots, x_{n}\right) .
$$

Proof. As

$$
\begin{aligned}
F_{\left(\left|X_{1}\right|, \ldots,\left|X_{n}\right|\right)}\left(x_{1}, \ldots, x_{n}\right) & =P_{\left(X_{1}, \ldots, X_{n}\right)}\left(\prod_{t=1}^{n}\left[-x_{t}, x_{t}\right]\right) \\
& \left.\left.=P_{\left(X_{1}, \ldots, X_{n}\right)}\left(\prod_{i=1}^{n}\right]-\infty, x_{i}\right] \cap\left(\overline{\bigcup_{t=1}^{n}\left(\mathbb{R}^{t-1} \times\right]-\infty,-x_{t}\left[\times \mathbb{R}^{n-t}\right)}\right)\right),
\end{aligned}
$$

then

$$
\begin{aligned}
& F_{\left(\left|X_{1}\right|, \ldots,\left|X_{n}\right|\right)}\left(x_{1}, \ldots, x_{n}\right) \\
& \left.\left.\left.\left.\quad=F_{\left(X_{1}, \ldots, X_{n}\right)}\left(x_{1}, \ldots, x_{n}\right)-P_{\left(X_{1}, \ldots, X_{n}\right)}\left(\bigcup_{t=1}^{n}\left(\prod_{i=1}^{t-1}\right]-\infty, x_{i}\right] \times\right]-\infty,-x_{t}\left[\times \prod_{i=t+1}^{n}\right]-\infty, x_{i}\right]\right)\right) .
\end{aligned}
$$

As, for every $t=1, \ldots, n$,

$$
\left.\left.\left.\left.\left.\left.\left(\prod_{i=1}^{n}\right]-\infty,-x_{i}\right]\right) \backslash\left\{\left(-x_{1}, \ldots,-x_{n}\right)\right\} \subset \bigcup_{t=1}^{n}\left(\prod_{i=1}^{t-1}\right]-\infty, x_{i}\right] \times\right]-\infty,-x_{t}\left[\times \prod_{i=t+1}^{n}\right]-\infty, x_{i}\right]\right)
$$

and $P\left(X_{1}=-x_{1}, \ldots, X_{n}=-x_{n}\right)=0$, then

$$
\begin{aligned}
& \left.\left.\left.\left.P_{\left(X_{1}, \ldots, X_{n}\right)}\left(\bigcup_{t=1}^{n}\left(\prod_{i=1}^{t-1}\right]-\infty, x_{i}\right] \times\right]-\infty,-x_{t}\left[\times \prod_{i=t+1}^{n}\right]-\infty, x_{i}\right]\right)\right) \\
& \left.\left.\geq P_{\left(X_{1}, \ldots, X_{n}\right)}\left(\left(\prod_{i=1}^{n}\right]-\infty,-x_{i}\right]\right) \backslash\left\{\left(-x_{1}, \ldots,-x_{n}\right)\right\}\right)=F_{\left(X_{1}, \ldots, X_{n}\right)}\left(-x_{1}, \ldots,-x_{n}\right) .
\end{aligned}
$$

So,

$$
F_{\left(\left|X_{1}\right|, \ldots,\left|X_{n}\right|\right)}\left(x_{1}, \ldots, x_{n}\right) \leq F_{\left(X_{1}, \ldots, X_{n}\right)}\left(x_{1}, \ldots, x_{n}\right)-F_{\left(X_{1}, \ldots, X_{n}\right)}\left(-x_{1}, \ldots,-x_{n}\right) .
$$

We recall the relation

$$
\left.F_{\left(\left|X_{1}\right|, \ldots,\left|X_{n}\right|\right)}\left(x_{1}, \ldots, x_{n}\right) \leq \prod_{t=1}^{n}\left[F_{|Z|}\left(\frac{x_{t}}{\alpha_{0}}\right)\right], \quad\left(x_{1}, \ldots, x_{n}\right) \in\right] 0,+\infty\left[^{n},\right.
$$

valid for a general $\operatorname{TGARCH}(p, q)$ (Gonçalves and Mendes-Lopes [3]) which may contribute to build bounds useful in practice, that is, only expressed in terms of the coefficients of the model and characteristics of the process $Z$. We point out that the statement in Theorem 3 of these upper and lower bounds of $F_{\left(X_{1}, \ldots, X_{n}\right)}\left(x_{1}, \ldots, x_{n}\right)$ when $\left.\left(x_{1}, \ldots, x_{n}\right) \in\right] 0,+\infty\left[^{n}\right.$ or $\left.\left(x_{1}, \ldots, x_{n}\right) \in\right]-\infty, 0\left[{ }^{n}\right.$ do not demand the weak stationarity of the process $X$. Nevertheless, to get some bounds useful in practice the 
weak stationarity of $X$ may be necessary. This is the case with lower bounds of $F_{\left(\left|X_{1}\right|, \ldots,\left|X_{n}\right|\right)}\left(x_{1}, \ldots, x_{n}\right)$ for some sub-families of these models deduced in Gonçalves and Mendes-Lopes [3].

\section{Application to Control Charts}

In problems related to assessing the performance of control charts for conditionally heteroskedastic processes with symmetric marginal distribution, it is important to evaluate the probabilities $P\left(\left|X_{t}\right| \leq x_{t}, t=1, \ldots, n\right)$ (Pawlak and Schmid [5]), concretely when evaluating the distribution of the run length (Severin and Schmid [6]). With this goal, upper and lower bounds for $P\left(\left|X_{t}\right| \leq x_{t}, t=1, \ldots, n\right)$ were established in Gonçalves and Mendes-Lopes [3] when $X$ is a $\operatorname{TGARCH}(p, q)$ process. In particular, if $X$ is a TARCH $(q)$ process and under general conditions, they conclude that the probability of no alarm until time $n$ in the in-control state, namely $P\left(\max _{1 \leq t \leq n} \frac{\left|X_{t}\right|}{\sigma_{X}} \leq x\right)$, can be evaluated, for certain values of $x$, using the independent generating process $Z$. From these results Gonçalves, Leite and MendesLopes [2] developed theoretical and simulated studies for control charts with symmetric limits. The lower and upper bounds obtained in Theorem 1 for the distribution function of $\left(X_{1}^{+},-X_{1}^{-}, \ldots, X_{n}^{+},-X_{n}^{-}\right)$are crucial to generalize this approach to control charts with asymmetric control limits, in the sense that, for $x, y \in \mathbb{R}$, the probability of no alarm until time $n$ in the in-control state is

$$
\begin{aligned}
& P\left(\min _{1 \leq t \leq n} X_{t}^{-} \geq x, \max _{1 \leq t \leq n} X_{t}^{+} \leq y\right) \\
& \quad=P\left(X_{t}^{-} \geq x, t=1, \ldots, n, X_{t}^{+} \leq y, t=1, \ldots, n\right) \\
& \quad=P\left(-X_{t}^{-} \leq-x, t=1, \ldots, n, X_{t}^{+} \leq y, t=1, \ldots, n\right) \\
& \quad=F_{\left(X_{1}^{+},-X_{1}^{-}, \ldots, X_{n}^{+},-X_{n}^{-}\right)}(y,-x, \ldots, y,-x)
\end{aligned}
$$

These asymmetric limits became relevant when the marginal distribution of the generating process is skewed, as a symmetric marginal distribution of the generating process necessarily leads a symmetric marginal distribution of the conditionally heteroskedastic process.

For example, if $p=q=1$ and $x<0$ and $y>0$ we obtain the following bounds for the probability of no alarm until time $n$ in the in-control state

$$
\begin{aligned}
& \prod_{t=1}^{n}\left[F_{Z}\left(\frac{y}{w_{t}}\right)-F_{Z}\left(\frac{x}{w_{t}}\right)\right] \\
& \quad \leq P\left(\min _{1 \leq t \leq n} X_{t}^{-} \geq x, \max _{1 \leq t \leq n} X_{t}^{+} \leq y\right) \leq\left[F_{Z}\left(\frac{y}{\theta}\right)-F_{Z}\left(\frac{x}{\theta}\right)\right]^{n}
\end{aligned}
$$


where $w_{t}=\alpha_{0}\left(1+\alpha_{1} y-\beta_{1} x\right) \frac{1-\gamma_{1}^{-1}}{1-\gamma_{1}}+\gamma_{1}^{t-1} E\left(\sigma_{1}\right)$ and $\theta=\alpha_{0}\left(1+\gamma_{1}\right)$. We note that, if $X$ becomes independent $\left(\alpha_{1}=\beta_{1}=\gamma_{1}=0\right)$, the equality holds for both bounds as we have $P\left(\min _{1 \leq t \leq n} X_{t}^{-} \geq x, \max _{1 \leq t \leq n} X_{t}^{+} \leq y\right)=\left[F_{Z}\left(\frac{y}{\alpha_{0}}\right)-F_{Z}\left(\frac{x}{\alpha_{0}}\right)\right]^{n}$.

\section{Conclusions}

The results here presented, namely the evaluation of the finite dimensional laws of the process $\left(X^{+},-X^{-}\right)$, lead us to the theoretical analysis of no alarm until time $n$ in the in-control state in a way that is not necessarily symmetrical.

Moreover, the results established in Section 2 were also used to bound the laws of the process taking into mind its application to control charts with unilateral control limits. The theoretical development of this study, particularly in the context of control charts, as well as its evaluation with simulated and real data are planned for future work. The generalization of these results to other models, for example with several thresholds or random thresholds, is also an open question with relevance in financial time series.

Acknowledgement. We thank the referees for their constructive suggestions.

\section{References}

1. Gonçalves, E., Leite, J., Mendes-Lopes, N.: Sobre as leis marginais de processos GARCH com limiares. In: Ferrão, M., Nunes, C., Braumann, C. (eds.) Estatística - Ciência Interdisciplinar, Actas do XIV Congresso da SPE, pp. 445-454. Edições SPE (2007)

2. Gonçalves, E., Leite, J., Mendes-Lopes, N.: The ARL of modified Shewhart control charts for conditionally heteroskedastic models. Stat. Papers (2011) doi: 10.1007/s00362-011-0408-Z

3. Gonçalves, E., Mendes-Lopes, N.: On the distribution of generalized threshold ARCH stochastic processes. Int. J. Pure Appl. Math. 35, 397-419 (2007)

4. Malmsten, H., Terasvirta, T.: Stylized facts of financial time series and three popular models of volatility. Eur. J. Pure Appl. Math. 3, 443-477 (2010)

5. Pawlak, M., Schmid, W.: On the distributional properties of GARCH processes. J. Time Ser. Anal. 22, 339-352 (2001)

6. Severin, T., Schmid, W.: Monitoring changes in GARCH processes. Allg. Stat. Arch. 83, 281307 (1999)

7. Taylor, S.J.: Asset price dynamics, volatility and prediction. Princeton University Press, Princeton (2007) 